\title{
Модель контакта двумерного металла и графеноподобного соединения с учетом их взаимодействия
}

\author{
(C) С.Ю. Давыдов ${ }^{1}$, О.В. Посредник ${ }^{2}$ \\ ${ }^{1}$ Физико-технический институт им. А.Ф. Иоффе Российской академии наук, \\ 194021 Санкт-Петербург, Россия \\ ${ }^{2}$ Санкт-Петербургский государственный электротехнический университет (ЛЭТИ), \\ 197376 Санкт-Петербург, Россия \\ E-mail: Sergei_Davydov@mail.ru
}

Поступила в Редакцию 25 ноября 2020 г.

В окончательной редакции 18 марта 2021 г.

Принята к публикации 19 марта 2021 г.

С учетом взаимодействия между слоями рассмотрена система графеноподобное соединение (GLC)-двумерный $d$-металл (2DM). В рамках диагонального приближения получены функции Грина слоев. Подробно изучен режим слабой связи GLC-2DM, для которого получены аналитические выражения для поправок к числам заполнения, переходу заряда и высоте барьера Шоттки. Показано, что найденные поправки наиболее существенны, когда в контакте участвуют металлы начала и конца $d$-рядов.

Ключевые слова: функция Грина, закон дисперсии, плотность состояний, переход заряда, барьер Шоттки.

DOI: 10.21883/FTP.2021.07.51020.9559

\section{1. Введение}

В последнее время возник интерес к двумерным металлам (2DM) [1-5]. Исходя из перспективы приборного использования 2DM представляется логичным рассмотреть систему контакта монослоев металла и изолятора (полупроводника). В простейшем случае в отсутствие взаимодействия между слоями задача о контакте двумерных $d$-металлов с графеноподобными соединениями (GLC) рассматривалась нами в [6]. Здесь мы это взаимодействие учтем. В отличие от работ [1-5], где в расчетах использовались различные варианты DFT (density functional theory), мы прибегнем к помощи достаточно простых моделей. В рамках таких моделей мы получим общие выражения для плотностей состояний взаимодействующих монослоев, рассмотрим режимы сильной и слабой связи 2DM-GLC и переход заряда между монослоями при различном взаиморасположении зон 2DM и GLC, приведем оценки высоты барьера Шоттки для контакта двумерного гексагонального нитрида бора с 2DM. Основная задача работы состоит в том, чтобы выяснить, как учет взаимодействия влияет на эти характеристики.

\section{2. Модель: общие соотношения}

В соответствии с [1] для 2DM устойчивой является гексагональная плотная упаковка, или треугольная решетка. Такой решетке отвечает закон дисперсии вида

$$
\begin{gathered}
E_{\mathrm{M}}(\mathbf{k})=\varepsilon_{m}-2 t_{m} f_{m}(\mathbf{k}), \\
f_{m}(\mathbf{k})=\cos \left(k_{x} a_{m}\right)+2 \cos \left(k_{x} a_{m} / 2\right) \cos \left(\sqrt{3} k_{y} a_{m} / 2\right),
\end{gathered}
$$

где $\omega-$ энергетическая переменная, $\varepsilon_{m}-$ энергия центра зоны, $t_{m}$ - энергия перехода электрона между соседними атомами металла, $\mathbf{k}=\left(k_{x}, k_{y}\right)-$ волновой вектор для движения электрона в плоскости $(x, y)$ листа, $a_{m}-$ расстояние между ближайшими соседями (б.с.) [6,7]. Тогда функция Грина свободного листа 2DM $g_{\mathrm{M}}(\omega, \mathbf{k})$ может быть представлена в виде $g_{\mathrm{M}}^{-1}(\omega, \mathbf{k})=\omega-E_{\mathrm{M}}(\mathbf{k})+i 0^{+}$. В Приложении (п. 1.) показано, что при учете взаимодействия 2DM c GLC в диагональном приближении функция $g_{\mathrm{M}}^{-1}(\omega, \mathbf{k})$ преобразуется в функцию Грина

$$
G_{M}^{-1}(\omega, \mathbf{k})=\omega-E_{M}(\mathbf{k})-\Lambda_{M}(\omega)+i \Gamma_{M}(\omega) .
$$

Здесь функция уширения $\Gamma_{\mathrm{M}}(\omega)=\pi V^{2} \rho_{\mathrm{GLC}}(\omega)$, где $\rho_{\mathrm{GLC}}(\omega)-$ энергетическая плотность состояний $\mathrm{GLC}, \quad V-$ матричный элемент взаимодействия 2DM-GLC; функция сдвига $\Lambda_{\mathrm{M}}(\omega)=$ $=V^{2} P \int_{-\infty}^{\infty} \rho_{\mathrm{GLC}}\left(\omega^{\prime}\right)\left(\omega-\omega^{\prime}\right)^{-1} d \omega^{\prime}$, где $P-$ символ главного значения. В низкоэнергетическом пределе плотность состояний для одной проекции спина одного электрона равна

$$
\rho_{\mathrm{GLC}}(\omega)= \begin{cases}2|\omega| / \xi^{2}, & \Delta \leq|\omega| \leq R \\ 0, & |\omega|<\Delta,|\omega|>R\end{cases}
$$

где $R=\sqrt{\xi^{2}+\Delta^{2}} \geq|\omega| \geq \Delta, \xi=\sqrt{2 \pi \sqrt{3}} t, t$ - энергия перехода между б.с. в GLC, $2 \Delta=E_{g}$ - ширина запрещенной зоны, начало отсчета энергии помещено в середину запрещенной зоны [8]. В [9] показано, что такой плотности состояний отвечает функция сдвига

$$
\Lambda_{\mathrm{M}}(\omega)=\frac{2 \omega V^{2}}{\xi^{2}} \ln \left|\frac{\omega^{2}-\Delta^{2}}{\omega^{2}-R^{2}}\right| .
$$

В свободном состоянии функция Грина GLC равна

$$
g_{\mathrm{GLC}}^{-1}(\omega, \mathbf{q})=\omega-E_{\mathrm{GLC}}^{ \pm}(\mathbf{q})+i 0^{+}
$$


и

$$
E_{\mathrm{GLC}}^{ \pm}(\mathbf{q})= \pm \sqrt{\Delta^{2}+t^{2} f_{\mathrm{GLC}}^{2}(\mathbf{q})},
$$

где в низкоэнергетическом приближении $f_{\mathrm{GLC}}(\mathbf{q})=$ $=3|\mathbf{q}| a / 2$ и волновой вектор $\mathbf{q}$ отсчитывается от точки $K_{\mathrm{GLC}}=a^{-1}(2 \pi / 3 \sqrt{3}, 2 \pi / 3)$ зоны Бриллюэна, $a-$ pacстояние между б.с. В Приложении (п. 1.) показано, что функция Грина GLC, взаимодействующего с 2DM, имеет вид

$$
G_{\mathrm{GLC}}^{-1}(\omega, \mathbf{q})=\omega-E_{\mathrm{GLC}}^{ \pm}(\mathbf{q})-\Lambda_{\mathrm{GLC}}(\omega)+i \Gamma_{\mathrm{GLC}}(\omega) .
$$

Здесь $\Gamma_{\mathrm{GLC}}(\omega)=\pi V^{2} \rho_{\mathrm{M}}(\omega)$, где

$$
\rho_{\mathrm{M}}(\omega)=-\pi^{-1} \operatorname{Im} \sum_{\mathbf{k}} g_{\mathrm{M}}(\omega, \mathbf{k})
$$

и

$$
\Lambda_{\mathrm{GLC}}(\omega)=V^{2} P \int_{-\infty}^{\infty} \rho_{\mathrm{M}}\left(\omega^{\prime}\right)\left(\omega-\omega^{\prime}\right)^{-1} d \omega^{\prime} .
$$

Отметим, что именно функции уширения $\Gamma_{M}(\omega)$, $\Gamma_{\mathrm{GLC}}(\omega)$ и сдвига $\Lambda_{\mathrm{M}}(\omega), \Lambda_{\mathrm{GLC}}(\omega)$ описывают взаимосвязь входящих в контакт монослоев GLC и 2DM.

Точное выражение для плотности состояний $\rho_{\mathrm{M}}(\omega)$, приведенное в [7], не дает возможности получить выражение для $\Lambda_{\mathrm{GLC}}(\omega)$ в аналитическом виде. Поэтому прибегнем к низкоэнергетической аппроксимации спектра (1). Рассматривая предел $f_{M}(\mathbf{k} \rightarrow 0)$, с точностью до $\left(k a_{m}\right)^{2}$ получим $E_{\mathrm{M}}(\mathbf{k}) \approx \varepsilon_{m}-6 t_{m}+6 t_{m}\left(k a_{m}\right)^{2}$. В [10] показано, что параболическому спектру соответствует постоянная (в определенном энергетическом интервале) плотность состояний. Поэтому для $d$-зоны шириной $W_{d}$ плотность состояний, приходящуюся на одну проекцию спина одного электрона, положим равной

$$
\rho_{\mathrm{M}}(\omega)= \begin{cases}1 / W_{d}, & \left|\omega-\varepsilon_{m}\right| \leq W_{d} / 2, \\ 0, & \left|\omega-\varepsilon_{m}\right|>W_{d} / 2,\end{cases}
$$

что соответствует модели Фриделя [11] ${ }^{1}$. Отсюда получаем функцию сдвига

$$
\Lambda_{\mathrm{LGC}}(\omega)=\frac{V^{2}}{W_{d}} \ln \left|\frac{\omega-\varepsilon_{m}+W_{d} / 2}{\omega-\varepsilon_{m}-W_{d} / 2}\right| .
$$

Для дальнейшего нам понадобятся энергетические плотности состояний $\tilde{\rho}_{\mathrm{M}}(\omega)$ и $\tilde{\rho}_{\mathrm{GLC}}(\omega)$, равные

$$
\begin{gathered}
\tilde{\rho}_{\mathrm{M}}(\omega)=-\pi^{-1} \operatorname{Im} \sum_{\mathbf{k}} G_{\mathrm{M}}(\omega, \mathbf{k}), \\
\tilde{\rho}_{\mathrm{GLC}}(\omega)=-\pi^{-1} \operatorname{Im} \sum_{\mathbf{q}} G_{\mathrm{GLC}}(\omega, \mathbf{q}) .
\end{gathered}
$$

\footnotetext{
${ }^{1}$ В модели Фриделя плотность состояний для всей $d$-зоны задается в виде $10 / W_{d}$. Для плотности состояний $s$-зоны можно написать аналогичное выражение, заменив 10 на $2, W_{d}$ на $W_{s}$ и $\varepsilon_{m}$ на $\varepsilon_{m}^{\prime}$. Так как $W_{s} \gg W_{d}$, вкладом $s$-зоны будем пренебрегать. Следует отметить, что низкоэнергетические аппроксимации спектров 2DM и GLC приводят к исчезновению особенностей ван Хова. В дальнейшем, однако, нас будут интересовать интегральные, а не дифференциальные характеристики, что делает такое упрощение приемлемым.
}

С учетом характера принятой нами аппроксимации $\tilde{\rho}_{\mathrm{M}}(\omega)$ можно приближенно представить в виде

$$
\tilde{\rho}_{\mathrm{M}}(\omega)=\frac{1}{\pi W_{d}}\left(\operatorname{arctg} \frac{\Omega_{\mathrm{M}}+W_{d} / 2}{\Gamma_{\mathrm{M}}(\omega)}-\operatorname{arctg} \frac{\Omega_{\mathrm{M}}-W_{d} / 2}{\Gamma_{\mathrm{M}}(\omega)}\right),
$$

где $\Omega_{\mathrm{M}}=\omega-\varepsilon_{m}-\Lambda_{\mathrm{M}}(\omega)$. При $\Gamma_{\mathrm{M}}(\omega)=0$ выражение (10) переходит в (7).

Общее выражение для плотности состояний эпитаксиального GLC приведено в $[8]$, откуда для $\tilde{\rho}_{\mathrm{GLC}}(\omega)$ при $R \leq \min \left\{\varepsilon_{m} \pm W_{d} / 2\right\}$, т. е. когда плотность состояний GLC перекрывается с плотностью состояний 2DM, получим

$$
\begin{gathered}
\tilde{\rho}_{\mathrm{GLC}}(\omega)=\frac{2 I(\omega)}{\xi^{2}}, \\
I(\omega)=\frac{\Gamma_{\mathrm{GLC}}(\omega)}{2 \pi} \ln \frac{\left|\xi^{4}+b \xi^{2}+c\right|}{c}+\frac{\left|\Omega_{\mathrm{GLC}}\right|}{\pi} \\
\times\left(\operatorname{arctg} \frac{2 \xi^{2}+b}{4 \Gamma_{\mathrm{GLC}}(\omega)\left|\Omega_{\mathrm{GLC}}\right|}-\operatorname{arctg} \frac{b}{4 \Gamma_{\mathrm{GLC}}(\omega)\left|\Omega_{\mathrm{GLC}}\right|}\right),
\end{gathered}
$$

где $b=2\left[\Gamma_{\mathrm{GLC}}^{2}(\omega)-\Omega_{\mathrm{GLC}}^{2}\right], c=\left(\Omega_{\mathrm{GLC}}^{2}-\Delta^{2}\right)^{2}+\Gamma_{\mathrm{GLC}}^{2}(\omega)$ $\times\left(\Gamma_{\mathrm{GLC}}^{2}(\omega)+2 \Delta^{2}+2 \Omega_{\mathrm{GLC}}^{2}\right), \Omega_{\mathrm{GLC}}=\omega-\Lambda_{\mathrm{GLC}}(\omega)$. При $\left|\omega-\varepsilon_{m}\right|>W_{d} / 2$, когда $\Gamma_{\mathrm{GLC}}(\omega)=0$, выражение (11) переходит в

$$
\tilde{\rho}_{\mathrm{GLC}}(\omega)= \begin{cases}2\left|\Omega_{\mathrm{GLC}}\right| / \xi^{2}, & \Delta \leq\left|\Omega_{\mathrm{GLC}}\right| \leq R, \\ 0, & \left|\Omega_{\mathrm{GLC}}\right|<\Delta,\left|\Omega_{\mathrm{GLC}}\right|>R .\end{cases}
$$

Это же выражение справедливо для случая $R>\max \left\{\varepsilon_{m} \pm W_{d} / 2\right\}, \quad$ когда плотность состояний GLC не перекрывается с плотность состояний 2DM. При $\Gamma_{\mathrm{GLC}}(\omega)=0$ выражение (12) переходит в (11).

\section{3. Режимы сильной и слабой связи 2DM-GLC}

В отсутствие взаимодействия энергетическая диаграмма контакта 2DM-GLC для некоторых случаев взаиморасположения зон изображена на рис. 1. Учет взаимодействия между компонентами контакта изменяет эту диаграмму. Для иллюстрации полученных выше общих результатов рассмотрим два предельных случая: режимы сильной $\left(V \gg \max \left\{t, t_{\mathrm{M}}, \Delta\right\}\right)$ и слабой $\left(V \ll \min \left\{t, t_{\mathrm{M}}, \Delta\right\}\right)$ связи $2 \mathrm{DM}-\mathrm{GLC}$. Как следует из (3) и (10), режим сильной связи для 2DM осуществляется при выполнении неравенства $\pi V^{2} \Delta / \xi^{2} \gg W_{d} / 2$ в интервале $\Delta \leq|\omega| \leq R$, откуда вместо невозмущенной плотности состояний $\rho_{\mathrm{M}}(\omega)=1 / W_{d}$ (см. (7)) получаем $\tilde{\rho}_{\mathrm{M}}(\omega) \approx \xi^{2} / 2 \pi^{2} V^{2} \Delta$. Таким образом, в режиме сильной связи взаимодействие приводит к резкому уменьшению плотности состояний 2DM в области сплошного спектра. При этом, однако, появляются локальные уровни, определяемые корнями уравнения $\omega-\varepsilon_{m}-\Lambda_{\mathrm{M}}(\omega)=0$ в области энергий $|\omega| \gg \max \left\{R, \varepsilon_{m} \pm W_{d} / 2\right\}$. Используя (4), получим 


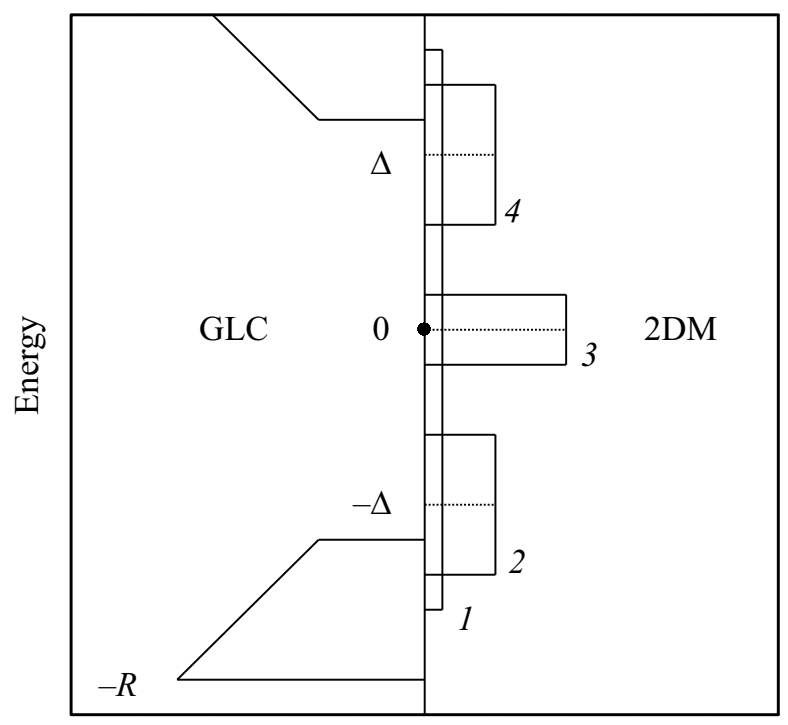

Density of states

Рис. 1. Энергетическая диаграмма контакта GLC-2DM без учета взаимодействия. Изображены случаи следующего взаиморасположения $d$-зоны 2DM и зон GLC: 1 - полное перекрытие $d$-зоной запрещенной зоны $(-\Delta, \Delta), 2,4$ - частичное перекрытие $d$-зоной запрещенной зоны и валентной зоны (зоны проводимости), 3 - встраивание $d$-зоны в запрещенную зону (изображен случай, когда $\varepsilon_{m}=0$ ). Штриховой линией обозначены положения центров $d$-зон $\varepsilon_{m}$, жирная точка соответствует нулевой энергии, энергия дна валентной зоны GLC равна $-R$, верхний срез рисунка имитирует уровень вакуума. Масштабы плотностей состояний GLC и 2DM произвольны.

уровни $\omega_{ \pm}^{*} \approx \pm V$, плотность состояний которых равна $\tilde{\rho}_{\mathrm{M}}^{ \pm}=v_{\mathrm{M}}^{ \pm} \delta\left(\omega-\omega_{ \pm}^{*}\right)$, где $v_{\mathrm{M}}^{ \pm}=\left|1-\partial \Lambda_{\mathrm{M}}(\omega) / \partial \omega\right|_{\omega_{ \pm}^{*}}^{-1}$ и $\delta(\ldots)-\delta$-функция Дирака $[12,13]$. Как показано в [9], при $V \rightarrow \infty$ имеем $v_{\mathrm{M}}^{ \pm} \rightarrow 1$. Таким образом, в пределе сильной связи электрон из зонного состояния 2DM переходит на лежащий ниже границы сплошного спектра глубокий локальный уровень.

Случай слабой связи в интервале энергий $\Delta \leq|\omega| \leq R$ реализуется при выполнении неравенства вида $2 \pi V^{2} R / \xi^{2} \ll \min \left\{\left|\Omega_{\mathrm{M}} \pm W_{d} / 2\right|\right\}$. Тогда вместо выражения (10) получим

$$
\begin{gathered}
\tilde{\rho}_{\mathrm{M}}(\omega) \approx \tilde{\rho}_{\mathrm{M}}^{\prime} \equiv \frac{1}{W_{d}}\left(1-\frac{2 V^{2} R W_{d}}{\xi^{2}\left[\left(W_{d} / 2\right)^{2}-\Omega_{\mathrm{M}}^{2}\right]}\right), \\
\left|\Omega_{\mathrm{M}}\right|<W_{d} / 2, \quad \Delta \leq|\omega| \leq R, \\
\tilde{\rho}_{\mathrm{M}}(\omega) \approx \tilde{\rho}_{\mathrm{M}}^{\prime \prime} \equiv \frac{1}{W_{d}} \frac{4\left|\Omega_{\mathrm{M}}\right| V^{2} R}{\xi^{2}\left[\Omega_{\mathrm{M}}^{2}-\left(W_{d} / 2\right)^{2}\right]}, \\
\left|\Omega_{\mathrm{M}}\right|>W_{d} / 2, \quad \Delta \leq|\omega| \leq R .
\end{gathered}
$$

В интервалах $|\omega|<\Delta$ и $|\omega|>R$ имеем $\tilde{\rho}_{\mathrm{M}}(\omega) \approx W_{d}^{-1}$, если $\left|\Omega_{\mathrm{M}}\right|<W_{d} / 2$, и $\tilde{\rho}_{\mathrm{M}}(\omega)=0$, если $\left|\Omega_{\mathrm{M}}\right|>W_{d} / 2$. Из (13) и (14) следует, что слабое взаимодействие приводит лишь к малым поправкам к невозмущенной плотности состояний $\rho_{\mathrm{M}}(\omega)$.
Перейдем теперь к влиянию взаимодействия на плотность состояний GLC. Отметим, что выражение (11) в пределе бесконечно широкой металлической зоны, когда в соответствии с (8) $\Lambda_{\mathrm{M}}=0$, достаточно подробно исследовалось в работах $[8,11]$. Для рассматриваемой здесь модели с конечной шириной $d$-зоны оценки показывают, что в режиме сильной связи GLC-2DM, когда $\pi V^{2} / W_{d} \gg R$, получим $\tilde{\rho}_{\mathrm{GLC}}(\omega) \approx W_{d} / \pi^{2} V^{2}$ при $\left|\omega-\varepsilon_{m}\right| \leq W_{d} / 2$. Вместо линейной функции от энергии (3) в интервале $\left|\omega-\varepsilon_{m}\right| \leq W_{d} / 2$ имеем $\tilde{\rho}_{\mathrm{GLC}}(\omega)=\mathrm{const}$, откуда следует, что сильное взаимодействие с 2DM качественно изменяет плотность состояний GLC, приводя к ее резкому уменьшению в области сплошного спектра. При этом, однако, как и в случае 2DM, появляются локальные состояния, энергия которых определяется корнями уравнения $\omega-\Lambda_{\mathrm{GLC}}(\omega)=0$ в области $|\omega| \gg \max \left\{R, \varepsilon_{m} \pm W_{d} / 2\right\}$. Используя (8), вновь получаем локальные уровни $\omega_{ \pm}^{*} \approx \pm V$, плотность состояний которых равна $\tilde{\rho}_{\mathrm{GLC}}^{ \pm}=v_{\mathrm{GLC}}^{ \pm} \delta\left(\omega-\omega_{ \pm}^{*}\right)$, где $v_{\mathrm{GLC}}^{ \pm}=\left|1-\partial \Lambda_{\mathrm{GLC}}(\omega) / \partial \omega\right|_{\omega_{ \pm}^{*}}^{-1}$. Как и в случае 2DM, при $V \rightarrow \infty$ получаем $v_{\mathrm{GLCC}}^{ \pm} \rightarrow 1$, т.е. имеет место переход электрона из зонных состояний на глубокий локальный уровень.

В случае слабой связи, когда отношение $\pi V^{2} / W_{d}$ является малой энергетической величиной задачи, $\tilde{\rho}_{\mathrm{GLC}}(\omega) \sim 2\left|\Omega_{\mathrm{GLC}}\right| / \xi^{2}+O\left(\pi V^{2} / W_{d}\right) \quad$ в $\quad$ интервале $\Delta \leq\left|\Omega_{\mathrm{GLC}}\right| \leq R \quad$ и $\quad \tilde{\rho}_{\mathrm{GLC}}(\omega) \sim O^{\prime}\left(\pi V^{2} / W_{d}\right)$ вне этого интервала, где $O(x)$ и $O^{\prime}(x)$ - малые функции (порядка $x$ ). Таким образом, плотность состояний $\tilde{\rho}_{\mathrm{GLC}}(\omega)$ не испытывает качественной перестройки по сравнению с $\rho_{\mathrm{GLC}}(\omega)$ (3). Изменяется, однако, энергетический интервал ненулевых значений $\tilde{\rho}_{\mathrm{GLC}}(\omega)$ и возникает нелинейность зависимости от энергии, так как $\Omega_{\mathrm{GLC}}=\omega=-\Lambda_{\mathrm{GLC}}(\omega)$.

Таким образом, сильная связь GLC-2DM кардинально изменяет плотности состояний компонентов контакта: при $V \rightarrow \infty$ в области сплошного спектра плотности состояний стремятся к нулю, но при этом вне сплошного спектра возникают связывающее $(-V)$ и антисвязывающее $(+V)$ состояния, соответствующие, на языке теории адсорбции, так называемой модели двухатомной поверхностной молекулы [13]. Поэтому, для того чтобы сохранить свойства (зачастую уникальные) исходных компонентов контакта, и в то же время, сформировать единую двухслойную систему, требуется слабая вандер-ваальсова связь. Таким образом, имеет место то же требование, что и при построении вертикальных гетероструктур $[14,15]$. Поэтому в дальнейшем мы будем рассматривать слабую связь GLC-2DM.

\section{4. Переход заряда и барьер Шоттки}

При нулевой температуре число заполнения $n_{\mathrm{GLC}}$ (равное полусумме чисел заполнения образующих элементарную ячейку атомов [8]) и число заполнения $n_{\mathrm{M}}=n_{d}$ (заполнение внешних электронных оболочек 
задается формулой $d^{N-2} s^{2}$, где $N$ изменяется от 3 до 11 [11]) соответственно равны

$$
\tilde{n}_{\mathrm{GLC}}=2 \int_{-\infty}^{\mu} \tilde{\rho}_{\mathrm{GLC}}(\omega) d \omega, \quad \tilde{n}_{\mathrm{M}}=10 \int_{-\infty}^{\mu} \tilde{\rho}_{\mathrm{M}}(\omega) d \omega,
$$

где $\mu-$ химический потенциал системы GLC-2DM, положение которого определяется из условия сохранения числа электронов: $n_{\mathrm{GLC}}+n_{\mathrm{M}}=\tilde{n}_{\mathrm{GLC}}+\tilde{n}_{\mathrm{M}}$, где $n_{\mathrm{GLC}}$ и $n_{\mathrm{M}}$ - числа заполнения на один атом элементарной ячейки GLC и 2DM. Переход заряда между компонентами контакта в системе с взаимодействием определяется параметром $\tilde{Z}=\tilde{n}_{\mathrm{GLC}}-\tilde{n}_{\mathrm{M}}$. Так как в отсутствие взаимодействия переход заряда $Z=n_{\mathrm{GLC}}-n_{\mathrm{M}}$, то изменение перехода заряда при учете взаимодействия равно

$$
\delta \tilde{Z}=\tilde{Z}-Z=-2 \delta \tilde{n}_{\mathrm{M}},
$$

где учтено, что $\delta \tilde{n}_{\mathrm{GLC}}=-\delta \tilde{n}_{\mathrm{M}}$ и $\delta \tilde{n}_{\mathrm{GLC}(\mathrm{M})}=\tilde{n}_{\mathrm{GLC}(\mathrm{M})}$ $-n_{\mathrm{GLC}(\mathrm{M})}$. Приведем некоторые численные оценки.

В качества GLC рассмотрим гексагональный нитрид бора $(h$-BN) как наиболее изученное двумерное соединение III-V. Работу выхода $\phi_{h \text {-BN }}$ и ширину запрещенной зоны $2 \Delta$ свободного $h$-BN примем равными 4.6 и 6.0 эВ соответственно [16]. Полагая $h$-BN недопированным и совмещая центр запрещенной зоны с химическим потенциалом $\mu_{h \text {-BN }}=-\phi_{h \text {-BN }}$, определяем начало отсчета энергии. Согласно [8], $t=2.28$ эВ, так что $\xi=7.52$ эВ и $R=8.10$ эВ. Так как цель настоящей работе состоит в выяснении роли взаимодействия, то, в отличие от [6], здесь мы не будем конкретизировать контактирующие с $h$-BN металлы, ограничившись их диаграммами, представленными на рис. 1. Для режима слабой связи оценки значений $\delta \tilde{n}_{\mathrm{M}}$ приведены в Приложсении (п. 2.).

Полагая $\delta \tilde{n}_{\mathrm{M}}=A F$, где $A=20 V^{2} R / \xi^{2} W_{d}$, для диаграмм 1 и 2 рис. 1 получим:

$$
\begin{gathered}
F_{1}=\ln \frac{4 \mu^{2}-W_{d}^{2}}{4 R^{2}-W_{d}^{2}}, \quad-R \leq \mu \leq-W_{d} / 2, \\
F_{2}=\ln \frac{\left|W_{d}^{2}-4 \Delta^{2}\right|}{4 R^{2}-W_{d}^{2}}+\ln \frac{\left(W_{d}+2 \Delta\right)\left(W_{d}+2 \mu\right)}{\left|W_{d}-2 \Delta\right|\left(W_{d}-2 \mu\right)}, \\
-W_{d} / 2<\mu<W_{d} / 2, \\
F_{3}=\ln \frac{\left|W_{d}^{2}-4 \Delta^{2}\right|}{4 R^{2}-W_{d}^{2}}+2 \ln \frac{W_{d}+2 \Delta}{\left|W_{d}-2 \Delta\right|}+\ln \frac{4 \mu^{2}-W_{d}^{2}}{\left|W_{d}^{2}-4 \Delta^{2}\right|}, \\
W_{d} / 2 \leq \mu \leq R .
\end{gathered}
$$

Прежде всего отметим, что расходимости функции $F(\mu)$ при $\mu= \pm W_{d} / 2$ не являются следствием аппроксимации (7), так как скачки плотности состояний являются прямым следствием двухмерности гексагональной плотной упаковки, соответствующей треугольной решетке [7]. С другой стороны, расходимости $F(\mu)$ при $R^{2}=W_{d}^{2}$ или $\Delta^{2}=W_{d}^{2}$ являются чисто случайными. Для наглядности функция $F(\mu)$ представлена на рис. 2.

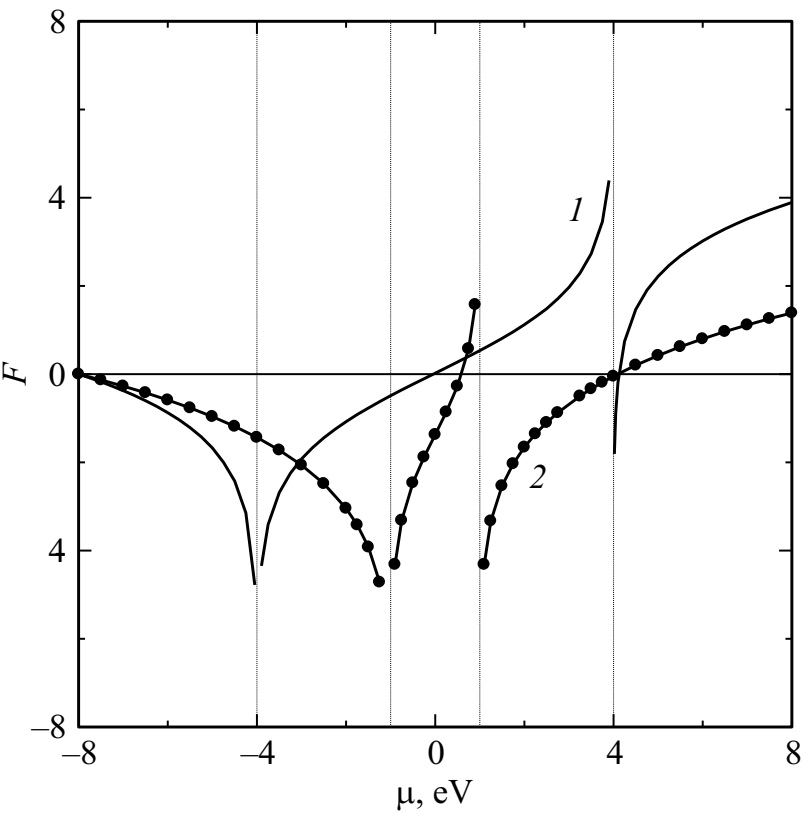

Рис. 2. Зависимость функции $F=\delta \tilde{n}_{\mathrm{M}} / A$, где $A=$ $=20 V^{2} R / \xi^{2} W_{d}$, от химического потенциала $\mu$ при $R=8$ эВ, $\Delta=3$ эВ, $W_{d}=8$ эВ (1) и $W_{d}=2$ эВ (2) (отмечено кружками). Вертикальными штриховыми линиями изображены асимптоты, отвечающие краям $d$-зон.

Отметим также, что диаграмма 1 с известным допущением ${ }^{2}$ может быть отнесена к двумерным молибдену и вольфраму (т.е. металлам, близким к серединам $d$-рядов), для которых $W_{d}$ равно 8.48 и 7.49 эВ соответственно (см. [6]). С теми же оговорками диаграмме 2 (а также 3 и 4 ) можно сопоставить металлы начала и конца $d$-рядов [6]. Подчеркнем, что приведенные выше выкладки справедливы при $A=20 V^{2} R / \xi^{2} W_{d} \ll 1$, для чего необходимо выполнение неравенства $V^{2} / \xi^{2} \ll 1$. Если считать, что GLC и 2DM связаны ван-дер-ваальсовым взаимодействием $\left(V \sim 0.1\right.$ эВ), то $V^{2} / \xi^{2} \sim 10^{-4}$, так что параметр $A$ можно считать малым.

Рассмотрим теперь, как учет взаимодействия GLC-2DM влияет на высоту барьера Шоттки. Для перехода металл-полупроводник высота барьера Шоттки для электронов $\Phi_{b}^{n}=\phi_{m}-\chi+\Delta \phi_{m}$, где $\phi_{m}-$ работа выхода металла, $\chi-$ энергия сродства к электрону полупроводника и $\Delta \phi_{m}=-4 \pi e^{2} l Q_{m} / S_{m}-$ изменение работы выхода металла, $e-$ элементарный заряд, $2 l$ - толщина двойного электрического слоя, $Q_{m}$ - заряд металлического атома, $S_{m}$ - площадь интерфейса, приходящаяся на один атом металла [18]. Так как в нашем случае $Q_{m}=n_{\mathrm{M}}-\tilde{n}_{\mathrm{M}}$, для

\footnotetext{
2 Допущение состоит в том, что при выводе выражений (17)-(19) мы положили $\varepsilon_{m}=0$. Такое упрощение приемлемо, так как разброс значений работ выхода, необходимых для определения энергий $\varepsilon_{m}$, весьма велик [17]. Здесь и в [6] мы приравниваем работы выхода $\phi_{m}$ для $3 \mathrm{DM}$ и 2DM на том основании, что в методе сильной связи (не путать с режимом сильной связи GLC-2DM) положение центра зоны $\varepsilon_{m}$ (в отличие от ее ширины) не зависит от размерности структуры.
} 
изменения высоты барьера Шоттки, вызванного учетом взаимодействия GLC-2DM, получим

$$
\delta \Phi_{b}^{n}=4 e^{2} \delta \tilde{n}_{\mathrm{M}} / a_{m},
$$

где мы положили $S_{m}=\pi a_{m}^{2} \quad$ и $\quad l=a_{m}$. Полагая $a_{m} \sim 3 \AA[19]$, имеем $\delta \Phi_{b}^{n} \sim 20 \delta \tilde{n}_{\mathrm{M}}$ (эВ).

\section{5. Заключение}

Итак, в настоящей работе мы рассмотрели двухслойную систему GLC-2DM, учитывая взаимодействие между слоями. Отбросив недиагональные по волновым векторам вклады, мы получили функции Грина (2) и (6). В режиме слабой связи слоев GLC и 2DM мы нашли аналитические выражения для поправок к числам заполнения и, тем самым, к переходу заряда и высоте барьера Шоттки. Оказалось, что эти поправки наиболее существенны в ситуации, когда химический потенциал $\mu$ находится вблизи краев $\left(\varepsilon_{m}-W_{d} / 2, \varepsilon_{m}+W_{d} / 2\right) d$-зоны 2DM. Оценки работы [6] показали, что значения $\mu$ мало отличаются от значений химического потенциала $\mu_{\mathrm{M}}^{0}=E_{d}-\left(W_{d} / 10\right)\left(5-n_{\mathrm{M}}^{0}\right)$ изолированного 2DМ. Отсюда следует, что поправки на взаимодействие важны, когда в контакте участвуют металлы начала и конца $d$-рядов. Вместе с тем следует отметить, что поправки носят количественный характер. Поэтому в ситуации, когда экспериментальная информация о конкретной системе отсутствует, вполне допустимы игнорирующие взаимодействие экспресс-оценки, выполненные по схеме работы [6].

\section{Приложсение}

1. Гамильтониан системы, состоящей из взаимодействующих монослоев $a$ и $b$, имеет вид

$$
H=\sum_{\mathbf{k}} E_{a}(\mathbf{k}) a_{\mathbf{k}}^{+} a_{\mathbf{k}}+\sum_{\mathbf{q}} E_{b}(\mathbf{q}) b_{\mathbf{q}}^{+} b_{\mathbf{q}}+\sum_{\mathbf{k}, \mathbf{q}}\left(V_{\mathbf{k q}} a_{\mathbf{k}}^{+} b_{\mathbf{q}}+\text { h.c. }\right),
$$

где $E_{a}(\mathbf{k})$ и $E_{b}(\mathbf{q})$ - законы дисперсии в свободных слоях $a$ и $b, a_{\mathbf{k}}^{+}\left(a_{\mathbf{k}}\right)$ - оператор рождения (уничтожения) электрона слоя $a$ в блоховском состоянии $|\mathbf{k}\rangle, b_{\mathbf{q}}^{+}(b)-$ аналогичные операторы для электрона слоя $b$ в блоховском состоянии $|\mathbf{q}\rangle$, $V(\mathbf{k}, \mathbf{q})$ - матричный элемент взаимодействия состояний $|\mathbf{k}\rangle$ и $|\mathbf{q}\rangle$, h.c. - эрмитово-сопряженное слагаемое. Вводя функции Грина $g_{a}(\omega, \mathbf{k})=\left(\omega-E_{a}(\mathbf{k})+i 0^{+}\right)^{-1}$, $g_{b}(\omega, \mathbf{q})=\left(\omega-E_{b}(\mathbf{q})+i 0^{+}\right)^{-1}$ для невзаимодействующих слоев $a, b$ и используя уравнение Дайсона $[12,13]$, получим уравнения

$$
\begin{gathered}
G_{a a}(\mathbf{k}, \mathbf{k})=g_{a}(\mathbf{k}, \mathbf{k})+g_{a}(\mathbf{k}, \mathbf{k}) \sum_{\mathbf{q}} V(\mathbf{k}, \mathbf{q}) G_{a b}(\mathbf{q}, \mathbf{k}), \\
G_{b a}(\mathbf{q}, \mathbf{k})=g_{b}(\mathbf{q}, \mathbf{q}) \sum_{\mathbf{k}^{\prime}} V\left(\mathbf{q}, \mathbf{k}^{\prime}\right) G_{a a}\left(\mathbf{k}^{\prime}, \mathbf{k}\right),
\end{gathered}
$$

откуда, оставляя под знаком суммы в (П.3) только слагаемое с $\mathbf{k}^{\prime}=\mathbf{k}$ (диагональное приближение), находим

$$
G_{a a}(\mathbf{k}, \mathbf{k})=\left(g_{a}^{-1}(\mathbf{k}, \mathbf{k})-\sum_{\mathbf{q}}|V(\mathbf{k}, \mathbf{q})|^{2} g_{b}(\mathbf{q}, \mathbf{q})\right)^{-1} .
$$

Воспользовавшись известной формулой из теории функций комплексного переменного $\left(x+i 0^{+}\right)^{-1}=$ $=P x^{-1}-i \pi \delta(x)$, где $P-$ символ главного значения, $\delta(\ldots)-\delta$-функция Дирака, выражение (П.4) можно переписать в виде

$$
G_{a a}(\mathbf{k}, \mathbf{k})=\left(\omega-E_{a}(\mathbf{k})-\Lambda_{a}(\omega)+i \Gamma_{a}(\omega)\right)^{-1},
$$

где

$$
\begin{gathered}
\Lambda_{a}(\omega)=P \sum_{\mathbf{q}}|V(\mathbf{k}, \mathbf{q})|^{2} g_{b}(\mathbf{q}, \mathbf{q}), \\
\Gamma_{a}(\omega)=\pi \sum_{\mathbf{q}}|V(\mathbf{k}, \mathbf{q})|^{2} \delta\left(\omega-E_{b} g_{b}(\mathbf{q})\right) .
\end{gathered}
$$

Полагая $\left\langle|V(\mathbf{k}, \mathbf{q})|^{2}\right\rangle=V^{2}$, где $\langle\ldots\rangle$ означает усреднения по зонам Бриллюэна слоев $a$ и $b$, получим функции Грина (2) и (6).

2. Рассмотрим диаграмму 1 (рис. 1), где $\varepsilon_{m}=0$ и $\Delta<W_{d} / 2<R$. Вводя интегралы

$$
I^{\prime}(a, b)=10 \int_{a}^{b} \tilde{\rho}_{\mathrm{M}}^{\prime}(\omega) d \omega, I^{\prime \prime}(c, d)=10 \int_{c}^{d} \tilde{\rho}_{\mathrm{M}}^{\prime \prime}(\omega) d \omega,
$$

где плотности состояний определяются формулами (13) и (14), для чисел заполнения 2DM имеем:

$$
\begin{array}{r}
\text { 1) } \tilde{n}_{\mathrm{M} 1}=I^{\prime \prime}(-R, \mu) \text { при }-R \leq \mu \leq-W_{d} / 2 ; \\
\text { 2) } \tilde{n}_{\mathrm{M} 2}=I^{\prime \prime}(-R,-\Delta)+I^{\prime}(-\Delta, \mu) \\
\text { при }-W_{d} / 2<\mu<W_{d} / 2 ; \\
\text { 3) } \tilde{n}_{\mathrm{M} 3}=I^{\prime \prime}(-R,-\Delta)+I^{\prime}(-\Delta, \Delta)+I^{\prime \prime}(\Delta, \mu) \\
\text { при } W_{d} / 2 \leq \mu \leq R .
\end{array}
$$

Полагая для простоты $\Omega_{\mathrm{M}}=\omega$, для интегралов $I^{\prime}(a, b)$ и $I^{\prime \prime}(c, d)$ получим:

$$
\begin{gathered}
I^{\prime}(a, b)=I_{0}^{\prime}(a, b)+I_{1}^{\prime}(a, b), \quad I_{0}^{\prime}(a, b)=\frac{10(b-a)}{W_{d}}, \\
I_{1}^{\prime}(a, b)=-\frac{20 V^{2} R}{\xi^{2} W_{d}} \ln \left|\frac{\left(W_{d}-2 a\right)\left(W_{d}+2 b\right)}{\left(W_{d}+2 a\right)\left(W_{d}-2 b\right)}\right|, \quad \text { (П.9) } \\
I^{\prime \prime}(c, d)=\frac{20 V^{2} R}{\xi^{2} W_{d}} \ln \left|\frac{4 d^{2}-W_{d}^{2}}{4 c^{2}-W_{d}^{2}}\right| .
\end{gathered}
$$

Из выражений (П.6)-(П.8) получаем $\delta \tilde{n}_{\mathrm{M} 1}=I^{\prime \prime}(-R, \mu)$, $\delta \tilde{n}_{\mathrm{M} 2}=I^{\prime \prime}(-R,-\Delta)+I_{1}^{\prime}(-\Delta, \mu), \quad \delta \tilde{n}_{\mathrm{M} 3}=I^{\prime \prime}(-R,-\Delta)$ $+I_{1}^{\prime}(-\Delta, \Delta)+I^{\prime \prime}(\Delta, \mu)$. Тогда с учетом (П.9) и (П.10) приходим к формулам (17)-(19). Аналогичные выражения могут быть записаны и для диаграммы 2, если учесть, что в этом случае $W_{d} / 2<\Delta$. 


\title{
Конфликт интересов
}

Авторы заявляют, что у них нет конфликта интересов.

\section{Список литературы}

[1] J. Nevalaita, P. Koskinen. Phys. Rev. B, 97, 035411 (2018).

[2] J. Nevalaita, P. Koskinen. AIP Advances, 10, 065327 (2020).

[3] S. Ono. arXiv: 2007.06774.

[4] S. Ono. Sci. Rep., 10, Article number: 11810 (2020).

[5] T. Wang, M. Park, Q. Yu, J. Zhang, Y. Yang. Materials Today Advances, 8, 100092 (2020).

[6] С.Ю. Давыдов. ФТТ, 63, 817 (2021).

[7] T. Hanisch, B. Kleine, A. Ritzl, E. Müller-Hartmann. Annalen Physik, 4, 303 (1995).

[8] С.Ю. Давыдов. ФТТ, 58, 779 (2016).

[9] С.Ю. Давыдов. ФТП, 51, 226 (2017).

[10] T. Ando, A.B. Fowler, F. Stern. Rev. Mod. Phys., 54, 438 (1982).

[11] У. Харрисон. Электронная структура и свойства твердых тел (М., Мир, 1983).

[12] С.Ю. Давыдов, А.А. Лебедев, О.В. Посредник. Элементарное введение в теорию наносистем (СПб., Лань, 2014).

[13] С.Ю. Давыдов. Теория адсорбиии: метод модельных гамильтонианов (СПб., Изд-во СПбГЭТУ „ЛЭТИ“, 2013). twirpx.com/file/1596114/

[14] A.K. Geim, I.V. Grigorieva. Nature, 499, 419 (2013).

[15] И.В. Антонова. ФТП, 50, 67 (2016).

[16] S. Thomas, M.S. Manju, K.M. Ajith, S.U. Lee, M.A. Zaeem. Physica E, 123, 114180 (2020).

[17] В.С. Фоменко. Эмиссионные свойства материалов (Киев, Наук. думка, 1981).

[18] С.Ю. Давыдов. ФТТ, 46, 2138 (2004).

[19] Ч. Киттель. Введение в физику твердого тела (М., Наука, 1978).

Редактор Г.А. Оганесян

\section{Model for two-dimensional metal and graphene-like compound junction with the account of their interaction}

\author{
S.Yu. Davydov' ${ }^{1}$, O.V. Posrednik ${ }^{2}$ \\ ${ }^{1}$ loffe Institute, \\ 194021 St. Petersburg, Russia \\ ${ }^{2}$ St. Petersburg State Electrotechnical University (ETU), \\ 197376 St. Petersburg, Russia
}

Abstract With the account of interlayer interaction, system of graphene-like compound (GLC) and two-dimensional $d$-metal (2DM) is considered. Within the scope of the diagonal approach corresponding Green functions are obtained. Regime of GLC-2DM weak coupling is studied thoroughly and analytical expressions for the corrections to the occupation numbers, charge transfer and Schottky barrier height are fulfilled. It is demonstrated that the corrections obtained are of significant importance for the metals of the beginning and the end of the $d$-rows. 